\title{
Recursos Educacionais Abertos (Open Educational Resources)
}

\author{
Renato Luís de Souza Dutra* \\ Liane Margarida Rockenbach Tarouco** \\ Centro Interdisciplinar de Novas Tecnologias da Educação \\ Universidade Federal do Rio Grande do Sul
}

\begin{abstract}
Resumo:
Inspirado no sucesso do movimento de software livre e da iniciativa OpenCourseWare(OCW) do Massachusetts Institute of Technology, é cada vez mais é crescente no ambiente acadêmico, a idéia de disponibilizar cursos e conteúdos de forma livre e aberta. Os Recursos Educacionais Abertos (REA), mas conhecidos por sua sigla em inglês OER (Open Educational Resources) são recursos voltados para o ensino, aprendizagem e pesquisa, disponibilizados de forma livre e aberta para a comunidade acadêmica em geral. Entre estes recursos incluem-se os conteúdos digitais de aprendizagem, ferramentas de apoio o desenvolvimento e uso destes conteúdos, bem como demais recursos necessários para a disponibilização destes conteúdos e cursos de uma forma livre a aberta.
\end{abstract}

Palavras-chave: OER, Recursos Educacionais Abertos, Conteúdos Abertos, Objetos de Aprendizagem

\begin{abstract}
Inspired by Open Source and MIT OpenCourseWare movements academic institutions are increasingly adopting the idea of deliver open and free digital content. Open Educational Resources (OER) are educational resources for research, teaching and learning purposes, delivered in a way free and open for the academic community. These resources include learning digital content, support tools for development and use of digital content and other resources that support the delivering of these contents and courses in a free and open way.
\end{abstract}

Keywords: OER, Open Educational Resources, Open Content, Learning Objects

\section{1. Introdução}

O movimento de software livre, conhecido também como movimento FLOSS (Free Libre and Open Source Software), tem se consolidado ao longo dos últimos anos. Projetos de sucesso como o Linux, Apache, Mozila entre outros tem inspirado pesquisadores e educadores na idéia da liberdade de utilização dos diversos recursos que são utilizados para a disponibilização de cursos on-line.

\footnotetext{
* Doutorando em Informática na Educação e Mestre em Ciência da Computação pela UFRGS

** Doutora em Engenharia Elétrica pela USP e Mestre em Ciência da Computação pela UFRGS

V. 5 № 1, Julho, 2007
} 
Paralelamente instituições de ensino de renome mundial como o Massachusetts Institute of Technology (MIT), no intuito de seguir sua missão de ajudar a desenvolver a sociedade através do conhecimento, criaram iniciativas como MIT OpenCourseWare (MIT OCW), que visa oferecer o acesso on-line através da Internet, a cursos e conteúdos de forma livre e aberta para alunos da instituição, pesquisadores e quaisquer interessados ao redor do mundo.

Esta conjunção de fatores deu inicio a um processo nas diversas instituições acadêmicas ao redor do mundo no sentido de disponibilizar conteúdos abertos (open content), desenvolvidos de forma colaborativa, assim como se faz no desenvolvimento de software livre. Com o amadurecimento desta idéia e com o seguimento em outras universidades de iniciativas similares ao MIT, o escopo do movimento de conteúdos abertos cresceu para abranger os demais recursos educacionais digitais utilizados para ajudar no desenvolvimento e disponibilização destes conteúdos, surgindo assim os Recursos Educacionais Abertos.

Este artigo busca descrever os Rercursos Educacionais Abertos destacando os Conteúdos Abertos e suas licenças, os OpenCourseWares e os Sistemas de Gerenciamento de Aprendizagem de Código Aberto.

\section{Recursos Educacionais Abertos}

Os Recursos Educacionais Abertos (REA), são mais conhecidos por sua sigla em inglês OER (Open Educational Resources). Segundo Hilen (2006), REA são materiais educacionais digitais disponibilizados de forma livre e aberta para a comunidade acadêmica em geral, que os utilizam para o ensino, aprendizagem e pesquisa. Este termo apareceu pela primeira vez em uma conferência da UNESCO em 2002 (HILEN, 2006).

Os REA abrangem os Conteúdos de Aprendizagem ou seja, cursos, módulos de conteúdo, objetos de aprendizagem entre outros. Eles incluem também ferramentas para apoiar o desenvolvimento, uso, reuso, busca e organização de conteúdos, bem como Sistemas de Gerenciamento de Aprendizagem e ferramentas de autoria. E por último os REA contemplam os chamados recursos de implementação que abrangem licenças para a disseminação de materiais abertos, bem como recursos de localização de conteúdos (HILEN, 2006).

Johnstone (2005) citado por Hilen (2006) define os recursos de acordo com sua função no processo de ensino-aprendizagem onde ele inclui: Recursos de Aprendizagem que incluem módulos de conteúdos, objetos de aprendizagem, ferramentas de avaliação, comunidades de aprendizagem, etc.;Recursos para Apoiar Professores contemplando ferramentas e materiais que ajudam os professores a criar, adaptar e reutilizar OER, bem como outras ferramentas de suporte; Recursos para Garantia da Qualidade, que garantem a qualidade da educação e a qualidade das práticas educacionais.

Segundo Wiley (2005), o termo Open Educational Resources tem suas raízes nos primeiros esforços na padronização e conceituação dos objetos de aprendizagem. Com a evolução da utilização dos objetos de aprendizagem Wiley definiu em 1998 o conceito de Open Content e criou a Open Content License/Open Publication License, visando a popularização dos conceitos do movimento FLOSS aplicados ao desenvolvimento de conteúdos educacionais. Com a rápida disseminação da idéia de conteúdos abertos indo além do escopo educacional, em 2001 Larry Lessig e outros membros da escola de direito de Harvard, fundaram a Creative Commons e com ela um conjunto flexível de 
licenças. Nesta mesma época o Massachussets Institute of Technology (MIT) resolveu disponibilizar grande parte de seus cursos para livre ao público para fins acadêmicos.

Em 2002 a UNESCO patrocinou um evento voltado para a discussão da disponibilização de recursos educacionais de forma universal, cunhando o termo Recursos Educacionais Abertos, com a seguinte definição:

Open Educational Resources are defined as "technology-enabled, open provision of educational resources for consultation, use and adaptation by a community of users for non-commercial purposes." They are typically made freely available over the Web or the Internet. Their principal use is by teachers and educational institutions support course development, but they can also be used directly by students. Open Educational Resources include learning objects such as lecture material, references and readings, simulations, experiments and demonstrations, as well as syllabi ,curricula and teachers' guides. (UNESCO, 2005)

\subsection{Conteúdos Abertos (Open Content) e Movimento de Código Aberto}

Quando se fala em OER, a primeira idéia que se vem a cabeça são os conteúdos abertos. Isto é natural, pois se pensarmos em todos os recursos que compõem o contexto OER, os conteúdos abertos são seu principal componente, sendo que todos os demais recursos, tais como softwares de autoria, ferramentas de busca, sistemas de gerenciamento de aprendizagem, licenças de uso para conteúdos e softwares, etc., servem de alguma forma para dar apoio à disponibilização de conteúdos abertos. Em resumo, o que se convencionou chamar de OER hoje é na verdade uma evolução no que se iniciou como movimento de Conteúdos Abertos.

O termo Conteúdo Aberto, ou Open Content, como dito anteriormente, foi criado por David Wiley e teve como base o movimento de código aberto (FLOSS). Sua idéia era se inspirar nos principais pressupostos do movimento FLOSS para a criação de conteúdos digitais educacionais que seguissem os mesmos preceitos da liberdade de software, que significa ter a liberdade para executar, estudar, adaptar, redistribuir e distribuir suas adaptações ao software (Rosen, 2005; FSF, 2007).

Pensando no desenvolvimento de software no contexto FLOSS, Eric Raymond, um renomado desenvolvedor e participante ativo de desenvolvimentos do $\mathrm{GNU}^{1}$, dissertou sobre o modelo de desenvolvimento do Linux versus o modelo tradicional de desenvolvimento de software em seu livro The Cathredal and the Bazaar. Ele nominou o modelo tradicional de Catedral, onde o software é desenvolvido por uma estrutura hierárquica bastante definida, onde excelentes desenvolvedores trabalham isoladamente até que a versão final esteja pronta, assim como eram construídas as antigas catedrais. Em contraponto, ele expôs o modelo Bazar, onde não existe uma hierarquia bem definida e todos podem contribuir e "debugar" o que quiser, sendo que as versões são disponibilizadas sucessivas vezes assim que tiverem alguma contribuição, até se chegar a uma versão final, algo semelhante a um bazar onde se escolhe o que vai levar (Raymond, 2000).

\footnotetext{
${ }^{1}$ GNU - Projeto criado em 1984 por Richard Stallman com o objetivo de desenvolver um sistema operacional similar ao Unix nos termos do FLOSS. 
Dando prosseguimento a sua idéia, ele cunhou dezenove princípios principais onde destacam-se alguns deles:

- Cada bom trabalho de software se inicia pela tentativa de resolver um problema pessoal que incomoda o desenvolvedor, ou seja, no mundo FLOSS o desenvolvimento de software vem da necessidade dos programadores em novas funcionalidades em softwares existentes;

- Bons programadores sabem como escrever um programa. Grandes programadores sabem o que reescrever ou reutilizar. Não é necessário que se reescreva todo o código, porque um código existente serve de estrutura para um novo código com mais funcionalidades;

- Para resolver um problema interessante, inicie procurando por um problema que é interessante para você. A melhor abordagem é iniciar por uma solução pessoal de seu dia-a-dia e depois expandi-la, pois geralmente o problema se torna um típico problema de um grande número de usuários;

Estas premissas indicam que o grande motivador do movimento FLOSS é o interesse dos participantes em se resolver problemas em comum. Todos estes princípios e premissas de Raymond existentes no desenvolvimento de software FLOSS, podem ser aplicados ao desenvolvimento de Conteúdos Abertos (WILEY, 2007). Wiley (2007) sustenta que não se pode desenvolver conteúdos abetos sob a premissa de que todo bom trabalho ou software no contexto OER se inicia com uma pesquisa das necessidades dos usuários e sim, assim como no software FLOSS um bom OER se inicia na necessidade pessoal do professor de resolver seu problema em termos de disponibilizar seus conteúdos em formato digital (WILEY, 2007).

\subsection{Licenças para Conteúdos Abertos}

Para que se disponiblize conteúdos abertos de forma livre e aberta, é muito importante se preocupar com direitos de autoria e propriedade intelectual destes conteúdos. Pensando nisso foram criadas licenças de uso específicas para conteúdos abertos, com base na experiência FLOSS.

Os primeiros esforços para a transposição do modelo FLOSS para utilização em conteúdos educacionais foram feitos pelo Dr. David Wiley, pesquisador no desenvolvimento de Objetos de Aprendizagem (LIANG, 2005). Wiley cunhou o termo "Open Content" e também as primeiras licenças que visavam a disseminação, reutilização de desenvolvimento colaborativo de conteúdo educacionais digitais. Para tanto criou as licenças Open Content License e Open Publication License. Estas licenças não estão sendo mais utilizadas e seu criador sugere a utilização de outras licenças como a Creative Commons (LIANG, 2005). Segundo Wiley (2007), seu intuito era de incentivar o debate e a disponibilização de conteúdos educacionais abertos. Segundo Wiley (2007), diversos livros foram publicados com a licença OPL, mas atualmente a Creative Commons provê um melhor suporte para a publicação e disponibilização de conteúdos digitais no contexto dos REAs.

Entre as licenças utilizadas para a disponibilização de conteúdos educacionais digitais abertos poderíamos citar o Open Content License/Open Publication License, a GNU Free Documentation License e o Creative Commons. 


\subsubsection{Open Content License \& Open Publication License}

A Open Content License segue os mesmo princípios das licenças FLOSS porém contemplando grande variedade de conteúdos, onde "conteúdo" não está propriamente definido, permitindo que a licença seja aplicada para qualquer meio (LIANG, 2005).

Esta licença foi a primeira desenvolvida por Wiley e foi criada pensando nas necessidades acadêmicas visando possibilitar o compartilhamento de trabalhos (LIANG, 2005). Seguindo as mesmas premissas do GNU GPL, a Open Content License se baseia na liberdade para o usuário final, expressa em três graus de liberdade: o direito de fazer cópias, o direito de redistribuir o conteúdo e o direito de modificar o conteúdo.

A Open Publication License sucedeu a Open Content License, melhorando alguns pontos que Wiley entendia como insuficientes (WILEY, 2007). Esta licença assume que qualquer publicação que esteja sob ela é totalmente livre para reprodução, distribuição ou modificação. A Open Publication License prevê que trabalhos podem ser reproduzidos e distribuídos na sua totalidade ou em partes em qualquer tipo de mídia, onde qualquer publicação em formato de livro requer a correta citação do autor ou editor original (OPL, 2007; LIANG, 2005). A propriedade da licença é de seu autor ou projetista (OPL, 2007). Por ser uma das primeiras licenças criadas para conteúdos abertos tinha algumas deficiências, que segundo Liang (2004), são melhor resolvidas pelas licenças Creative Commons e GNU Publicationa License.

Um dos problemas destas licenças segundo Liang (2004) é que uma pessoa pode impor certas restrições que podem alterar na natureza da licença, transformando ela em uma licença não aberta. Isto pode ser feito por um pressuposto escrito em uma das sessões (sessão VI) da licença que permite ao licenciador adicionar novas cláusulas que podem sugerir que podem haver grandes modificações sem a devida permissão do licenciador. Isto faz com que praticamente existam dois tipos de licença dentro de uma, sendo confuso tanto para o licenciador como para o licenciado (LIANG, 2005). Segundo a visão do GNU (GNU, 2007), esta licença não pode ser considerada uma licença de documentação livre por não ser uma licença no modelo copyleft ${ }^{2}$, pois o conceito de copyleft se aplica somente se o detentor dos direitos não exerça nenhuma das opções de licença listada na seção VI (GNU, 2007).

\subsubsection{GNU Free Documentation License}

A GNU Free Documentation License é uma licença que objetiva ser utilizada em documentações abertas sob o modelo copyleft. Ela pode ser utilizada para a documentação de manuais e também para outros tipos de trabalhos como livros, dicionários, não se restringindo somente para trabalhos textuais (GNU, 2007).

Esta licença foi criada como complemento a licença GNU GPL (utilzada por softwares como o Linux) e teve como objetivo ser utilizada em manuais de softwares sob a licença GNU, documentos ou outros textos instrucionais que possuíssem algum "valor funcional" (LIANG, 2005). Devido a estas definições esta licença é considera

\footnotetext{
2 Copyleft - meio de utilizar a legislação de proteção dos direitos autorais visando retirar barreiras à utilização, distribuição e modificação de uma obra criativa devido à aplicação clássica das normas de Propriedade Intelectual, obrigando que as modificações do software, ou extensões do mesmo, sejam sob os mesmo termos do software original, ou seja FLOSS.
} 
tecnicamente confusa, não sendo recomendada para textos que não forem técnicos (LIANG, 2005).

\subsubsection{Creative Commons}

A Creative Common License é uma das iniciativas mais recentes e mais significantes em termos de licenças para conteúdos abertos, tendo em um período muito curto de tempo ganhado grande aceitação (LIANG, 2005). Amparado por licenças bem definidas e por uma grande quantidade de informações fornecidas em seu sítio, esta licença tem se tornado a mais utilizada por todas as pessoas interessadas em licenciar conteúdos dos mais variados tipos no abertos (LIANG, 2005). Isto ocorre porque estas licenças têm como objetivo facilitar o processo de se basear em obras de outros, possibilitando aos criadores a concessão para que outros se baseiem em sua obra original de forma fácil e segura, dispensando advogados ou especialistas em licenciamento, através de rótulos que estão diretamente ligados à versões de licenças legíveis eletronicamente (MRI) (LESSIG, 2005).

Inspirada no movimento FLOSS, a Creative Commnos se baseia no conceito de que é preciso criar e disponibilizar uma grande quantidade de informações e conteúdos, com vistas a assegurar a criatividade de forma sustentada. Para isto, se fazem necessários mecanismos que assegurem este objetivo, através de um conjunto de licenças que permitam conteúdos abertos e colaboração, bem como atuando como um repositório de conteúdos abertos (LIANG, 2005). Além disso, a Creative Commons colabora para educar as pessoas acerca dos direitos autorais, liberdade de expressão e comunicação e domínio público (LIANG, 2005)..

No Creative Commons, o criador pode combinar uma série de liberdades ou restrições, como por exemplo optar por uma licença que permita qualquer uso, pode também escolher uma licença somente para uso não comercial ou também optar por uma licença que permita qualquer utilização desde que estas liberdades sejam repassadas aos usos posteriores (copyleft) (LESSIG, 2005). Em resumo, estas opções criam um conjunto de liberdades que se sobrepõem à legislação de copyright, permitindo também liberdades que ultrapassam o uso legítimo tradicional, tudo isto de forma que os usuários não necessitem contratar um advogado para utilizar os conteúdos sob estas licenças, pois criam na verdade um conjunto plausível de legislação de copyright (LESSIG, 2005).

\subsection{OpenCouseWare}

Outro importante contribuídor na disseminação do conceito de OER são os Open Cousewares. As iniciativas de Open Coursewares (OpenCourseWare - OCW), hoje presentes em diversas universidades de renome ao redor do mundo, tiveram seu inicio em instituições americanas importantes, tendo como pioneiro o MIT. O OpenCourseWare do MIT (MIT OCW), começou a ser delineado quando o diretor do MIT Robert Brown solicitou posicionamento da instituição com relação a Educação a Distância através da Internet. Como resultado o MIT criou uma série de recomendações que serviram de idéia básica para o OCW, todas elas em consonância com os valores e a missão do MIT (MASSACHUSETTS INSTITUTE OF TECHNOLOGY, 2007). No dia 4 de abril de 2001, o presidente do MIT Charles Vest anunciou em o inicio do projeto MIT OCW (LONG, 2002).

O MIT OCW não é um conjunto de aulas on-line e sim um processo que tem como objetivo fazer com que o material utilizado na maioria dos cursos de graduação e pós- 
graduação, fique disponível de forma livre e on-line para qualquer usuário ao redor do mundo (LONG, 2002). Hoje, o MIT OCW é uma iniciativa em grande escala para a publicação de diversos cursos baseados na Web, provindos do corpo docente da MIT (OCW, 2007). Através desta iniciativa foi possível compartilhar de maneira aberta os conteúdos e cursos do MIT, com educadores, alunos matriculados e aprendizes ao redor do mundo (MASSACHUSETTS INSTITUTE OF TECHNOLOGY, 2007). Entre os recursos disponibilizados de maneira livre estão o syllabi, as notas de aula, os calendários de cursos, os arranjos de problemas para aulas com suas soluções, os exames, as listas de leitura, os vídeos de aulas, etc. Todos eles oriundos de 1550 cursos do MIT de 34 departamentos de todas as cinco Escolas da instituição (MASSACHUSETTS INSTITUTE OF TECHNOLOGY, 2007).

Com o sucesso do MIT OCW, outras universidades começaram projetos semelhantes, entre os qusis poderíamos citar a Utah State University OCW, Johns Hopkins School of Public Health OCW, Tufts University OCW, Foothill De-Anza SOFIA, Carnegie Mellon Open Learning Initiative, Japan Opencourseware Consortium(JOCW), entre outras.

\subsection{Sistemas de Gerenciamento de Aprendizagem de Código Aberto}

Os Sistemas de Gerenciamento de Aprendizagem (SGA) que no Brasil também são conhecidos como Ambientes Virtuais de Aprendizagem (AVA), são um importante componente dentro do contexto dos REAs, pois normalmente são através destes SGAs que os conteúdos são agregados em cursos e disponibilizados para a comunidade.

Para que estes SGAs. Estejam em consonância com as melhores práticas educacionais abertas, é recomendado que façam parte de projetos sob licenças FLOSS, tais como GNU GPL, Mozzila License, BSD License, entre outras. Isto porque para que a comunidade faça uso dos REA em sua totalidade, não deveria haver nenhum elemento que restrinja a liberdade do usuário de usar, modificar, copiar e redistribuir qualquer recurso dos REA. Entre os SGAs disponiblizados sob licenças FLOSS destacam-se Moodle, Claroline, Atutor, Ilias, Teleduc, entre outros.

No Brasil, o ponto de partida para o inicio da adoção da idéia de REA pode ser creditada à ênfase do governo atual na utilização de software FLOSS. No âmbito dos SGAs isto tem ganhado destaque a partir do advento da Universidade Aberta do Brasil (UAB). A UAB, através da organização de parcerias entre universidades por seu caráter público e capilaridade, torna crucial a utilização de alguns componentes dos REA. Para tanto, o SGA escolhido para a UAB foi o Moodle, que já é utilizado por diversas universidades no Brasil.

\subsection{Conclusões}

Os Recursos Educacionais Abertos são fruto do sucesso do movimento FLOSS, bem como na importante iniciativa do MIT em disponibilizar seus conteúdos digitais on-line de forma livre e aberta.. Cada vez mais se consolida a idéia de que as boas práticas educacionais abertas vão muito além do desenvolvimento de conteúdos abertos, se estendendo a todos os componentes necessários para implementar iniciativas similares ao do MIT.

Se pensarmos em termos de Brasil, os Recursos Educacionais Abertos podem ser um importante instrumento para a disseminação e universalização do conhecimento a partir 
de universidades públicas e privadas no Brasil. $O$ sucesso da utilização e desenvolvimento de softwares sob os preceitos do movimento FLOSS, nos permite projetar que a utilização de Recursos Educacionais Abertos, pode ser alternativa viável para se criar uma infra-estrutura que suporte o desenvolvimento colaborativo de conteúdos abertos e sua conseqüente disponibilização na forma de cursos ou objetos de aprendizagem. Estes cursos abertos, ou OCWs seriam entregues utilizando Sistemas de Gerenciamento de Aprendizagem open source, hoje largamente utilizados em universidades no Brasil.

\section{Referências:}

FREE SOFTWARE FOUNDATION. The Free Software Definition. Disponível em $<$ http://www.fsf.org/licensing/essays/free-sw.html>. Acesso em: 01 fev. 2007.

GNU. GNU's Not Unix! - Free Software, Free Society. Disponível em $<$ http://www.gnu.org/> . Acesso em: 10 fev. 2007.

HILEN, J. Open Educational Resources: Opportunities and Challenges. OECD's Centre for Educational Research and Innovation. Disponível em < http://www.oecd.org/dataoecd/5/47/37351085.pdf >. Acesso em: 15 dez. 2006.

LESSIG, L. Free Culture: How Big Media Uses Technology and the Law to Lock Down Culture and Control Creativity. New York: Penguin Group, 2005.

LIANG, L. Guide to open content licenses. Roterdam: Piet Zwart Institute, 2004.

LONG, P. D. OpenCourseWare: Simple Idea, Profound Implications. Eric Syllabus, v15 n6 p12-14,16. 2002.

MASSACHUSETTS INSTITUTE OF TECHNOLOGY. MIT's OpenCourseWare. Disponível em <http://ocw.mit.edu/index.html> . Acesso em: 01 mai. 2007.

ROSEN, L. Open Source License: Software Freedom and Intelectual Property Law. Upper Saddle River, NJ: Prentice Hall, 2005.

UNESCO. OPEN EDUCATIONAL RESOURCES OPEN CONTENT FOR HIGHER EDUCATION - Final Forum Report. Disponível em $<$ http://portal.unesco.org/ci/en/files/21713/11438000259OER Forum Final Report. pdf/OER\%2BForum\%2BFinal\%2BReport.pdf> . Acesso em: 10 fev. 2007.

WILEY, D. The Current State of Open Educational Resources. 2005 Disponível em

$<$ http://www.oecd.org/document/32/0,2340,en $2649 \quad 33723 \quad 36224352 \quad 1 \quad 1 \quad 11$ 1,00.ht ml>. Acesso em: 10 abr. 2007.

WILEY, D. Iterating toward openness Blog. Disponível em $<$ http://opencontent.org/blog/>. Acesso em 15 mai. 2007. 